\title{
iNOS Activity Modulates Inflammation, Angiogenesis, and Tissue Fibrosis in Polyether-Polyurethane Synthetic Implants
}

\author{
Puebla Cassini-Vieira, ${ }^{1,2}$ Fernanda Assis Araújo, ${ }^{3}$ \\ Filipi Leles da Costa Dias, ${ }^{1}$ Remo Castro Russo, ${ }^{2,4}$ Silvia Passos Andrade, ${ }^{1}$ \\ Mauro Martins Teixeira, ${ }^{2}$ and Luciola Silva Barcelos ${ }^{1,2}$ \\ ${ }^{1}$ Laboratório de Angiogênese e Células-Tronco, Departamento de Fisiologia e Biofísica, Universidade Federal de Minas Gerais, \\ 31270-901 Belo Horizonte, MG, Brazil \\ ${ }^{2}$ Laboratório de Imunofarmacologia, Departamento de Bioquímica e Imunologia, Universidade Federal de Minas Gerais, \\ 31270-901 Belo Horizonte, MG, Brazil \\ ${ }^{3}$ Laboratório de Angiogênese, Área de Ciências Fisiológicas-ARFIS, Universidade Federal de Uberlândia, \\ 38405-320 Uberlândia, MG, Brazil \\ ${ }^{4}$ Laboratório de Imunologia e Mecânica Pulmonar, Departamento de Fisiologia e Biofísica, 31270-901 Belo Horizonte, MG, Brazil
}

Correspondence should be addressed to Luciola Silva Barcelos; luciolasbarcelos@gmail.com

Received 12 January 2015; Revised 28 April 2015; Accepted 28 April 2015

Academic Editor: Jan G. C. van Amsterdam

Copyright (C) 2015 Puebla Cassini-Vieira et al. This is an open access article distributed under the Creative Commons Attribution License, which permits unrestricted use, distribution, and reproduction in any medium, provided the original work is properly cited.

\begin{abstract}
There is considerable interest in implantation techniques and scaffolds for tissue engineering and, for safety and biocompatibility reasons, inflammation, angiogenesis, and fibrosis need to be determined. The contribution of inducible nitric oxide synthase (iNOS) in the regulation of the foreign body reaction induced by subcutaneous implantation of a synthetic matrix was never investigated. Here, we examined the role of iNOS in angiogenesis, inflammation, and collagen deposition induced by polyether-polyurethane synthetic implants, using mice with targeted disruption of the iNOS gene (iNOS ${ }^{-/-}$) and wild-type (WT) mice. The hemoglobin content and number of vessels were decreased in the implants of $\mathrm{iNOS}^{-/-}$mice compared to WT mice 14 days after implantation. VEGF levels were also reduced in the implants of $\mathrm{iNOS}^{-/-}$mice. In contrast, the iNOS ${ }^{-/-}$implants exhibited an increased neutrophil and macrophage infiltration. However, no alterations were observed in levels of CXCL1 and CCL2, chemokines related to neutrophil and macrophage migration, respectively. Furthermore, the implants of $\mathrm{iNOS}^{-1-}$ mice showed boosted collagen deposition. These data suggest that iNOS activity controls inflammation, angiogenesis, and fibrogenesis in polyether-polyurethane synthetic implants and that lack of iNOS expression increases foreign body reaction to implants in mice.
\end{abstract}

\section{Introduction}

The implantation of polyether-polyurethane synthetic matrix has been used as a framework to induce fibrovascular tissue growth that has similarities with tissue formed in the physiological and pathological processes where angiogenesis, inflammation, and fibrogenesis are important concurrent processes. This model provides a chronically inflamed environment that mimics the ones that occur after mechanical tissue injuries such as in balloon angioplasty insertion, as well as during wound healing, inflammatory diseases, and foreign body reaction [1]. Indeed, at present, there is considerable interest in implantation techniques and scaffolds for tissue engineering and, for safety and biocompatibility reasons, inflammation, angiogenesis, and fibrosis need to be determined, as the success of the procedure is conditioned by an adequate inflammatory angiogenesis response to the device as well as by its degree of encapsulation $[1,2]$.

One of the main inflammatory mediators involved in both inflammation and angiogenesis is the nitric oxide (NO). $\mathrm{NO}$ can be synthesized by three different isoforms of NO synthase: neuronal (nNOS), endothelial (eNOS), and inducible (iNOS) synthases [3]. NO production due to cytokineinduced expression of inducible nitric oxide synthase (iNOS) 
is largely involved in the pathophysiology of inflammation [4-6]. Studies conducted in iNOS-deficient mice and/or under iNOS inhibition have provided evidence that inducible NO governs a broad spectrum of processes, such as recruitment and adhesion of leukocyte $[7,8]$, inflammatory diseases $[9,10]$, wound healing $[11,12]$, ischemia [13-15], and tumor-induced angiogenesis [16]. In addition, NO-releasing compounds have proved effective in attenuating foreign body reaction to subcutaneous implant $[17,18]$. While these studies have been decisive in demonstrating the potential activity of $\mathrm{NO}$ in minimizing the adverse foreign body reaction, we found no study that investigated the role of endogenous iNOS in the regulation of the foreign body reaction induced by subcutaneous implantation of synthetic matrix in mice.

We hypothesized that iNOS might modulate inflammatory angiogenesis in synthetic implants. Therefore, our aim was to study the effects of iNOS deletion on different features of the foreign body response induced by polyetherpolyurethane implants in terms of inflammation, neovascularization, and fibrogenesis in the synthetic matrix. We report that the deletion of iNOS was able to modulate critical features of inflammation, neovascularization, and collagen deposition on the fibrovascular tissue induced by sponge implants in mice as the absence of this enzyme leads to reduced angiogenesis and exacerbated inflammation and fibrosis in the synthetic implants.

\section{Material and Methods}

2.1. Animals. All animal care and experimental procedures complied with the guidelines established by our local Institutional Animal Welfare Committee. Efforts were made to avoid all unnecessary distress to animals. Male C57BL/ 6 mice aged 7-8 weeks (20-25 g body weight) with genetic deletion of iNOS (iNOS ${ }^{-/}$) and wild-type (WT) mice were provided by Dr. Leda Quércia Vieira (Department of Biochemistry and Immunology at the Institute of Biological Sciences, Federal University of Minas Gerais, Brazil). The animals were housed individually and provided with chow pellets and water ad libitum. The light/dark cycle was 12:12 h with lights on at 7:00 am and lights off at 7:00 pm.

2.2. Sponge Discs Implantation. Polyether-polyurethane sponge (Vitafoam Ltd., Manchester, UK) was used as the implanted material, as described previously $[2,19,20]$. The implants were in the shape of discs, $5 \mathrm{~mm}$ thick $\times 8 \mathrm{~mm}$ diameter. They were soaked overnight in $70 \% \mathrm{v} / \mathrm{v}$ ethanol and sterilized by boiling in distilled water for 15 minutes before implantation. The animals were anaesthetized with a mixture of ketamine $100 \mathrm{mg} / \mathrm{kg}$ and xylazine $10 \mathrm{mg} / \mathrm{kg}$ and the dorsal hair was shaved and the exposed skin wiped with $70 \%$ ethanol. The sponge discs were aseptically implanted into a subcutaneous pouch, which had been made with curved artery forceps through a $1-\mathrm{cm}$ long dorsal midline incision. Postoperatively, the animals were monitored for any signs of infection at the surgical site, discomfort, or distress; any animals showing such signs were promptly euthanized with anesthetic excess. The implants were evaluated 14 days after implantation to assess vascularization (hemoglobin content, cytokines levels, and histological analysis), inflammatory markers (MPO and NAG activities and cytokines levels), and collagen deposition (Picrosirius-red staining).

2.3. Hemoglobin Extraction and Measurement. The extent of vascularization of the sponge implants was assessed by the amount of hemoglobin $(\mathrm{Hb})$ detected in the tissue using the Drabkin method $[19,20]$. At 14 days after implantation, the animals were euthanized with an excess of anesthetic and the sponge implants were carefully removed, dissected, cleared of any adherent tissue, and weighed. Each implant was homogenized (Tekmar TR-10, OH) in $2 \mathrm{~mL}$ of Drabkin reagent (Labtest, São Paulo, Brazil) and centrifuged at $12000 \times \mathrm{g}$ for $20 \mathrm{~min}$. The supernatants were filtered through a $0.22-\mu \mathrm{m}$ Millipore filter. The hemoglobin concentration in the samples was determined spectrophotometrically by measuring absorbance at $540 \mathrm{~nm}$ using an ELISA plate reader and comparing it against a standard hemoglobin curve. Hemoglobin content in the implant was expressed as $\mu \mathrm{g}$ of $\mathrm{Hb}$ per mg wet tissue.

2.4. ELISA for Cytokines/Chemokines. The supernatants from centrifugation of sponge homogenates (see hemoglobin measurement method) were used to examine the levels of VEGF, CXCL1/KC, CCL2/MCP-1, TNF- $\alpha$, IL-10, and IFN$\gamma$ produced in sponge implants by ELISA. The assays were performed using kits from R\&D Systems and according to the manufacturer's instructions. Standards were 0.5$\log _{10}$ dilutions of recombinant murine cytokines from $7.5 \mathrm{pg} \mathrm{mL}^{-1}$ to $1000 \mathrm{pg} \mathrm{mL}^{-1}$. The threshold of sensitivity for each cytokine/chemokine was $7.5 \mathrm{pg} / \mathrm{mL}$. The results were expressed as pg cytokine per mg wet tissue.

2.5. Quantification of Neutrophil or Macrophage Tissue Accumulation. Pellets from centrifugation of sponge homogenates (see hemoglobin measurement method) were divided into two portions and suspended with different buffers specific for measurement of myeloperoxidase (MPO) or Nacetyl- $\beta$-D-glucosaminidase (NAG) activities used as neutrophil and macrophage accumulation indexes, respectively, as described previously [20].

2.6. Histological Analysis. The sponge implants from a separate group of mice were carefully excised, dissected free of adherent tissue, and fixed in formalin $(10 \% \mathrm{w} / \mathrm{v}$ in isotonic saline). Sections $(5 \mu \mathrm{m})$ were stained with hematoxylin and eosin (H\&E) or Picrosirius-red and processed for lightmicroscopic studies. To perform a morphometric analysis of blood vessels, cross section images obtained from 15 sequential fields $\left(8533 \mu \mathrm{m}^{2}\right)$ from each implant were captured with a plan apochromatic objective (40x) in light microscopy (final magnification $=400 \mathrm{x})$. A countable vessel was defined as a structure with a lumen whether or not it contained red blood cell [1]. For collagen analysis, images were obtained from 10 fields $\left(\right.$ area $\left.=343,592 \mu \mathrm{m}^{2}\right)$ at 20x (final magnification $=200 \mathrm{x}$ ) under polarized light (Olympus). The images were digitized through an Olympus BX43 with an Olympus Q-color 5 microcamera and morphometric analyses were performed 
on digital images using ImageProPlus 7.0 Software. A single observer blinded to the condition and treatment performed the analysis. Additionally, to standardize the image analysis, the fields were photographed on the same day to avoid any variability associated with the light source.

2.7. Statistical Analysis. The results were expressed as mean \pm SEM. Statistical comparisons between two groups of mice were carried out using Student's $t$-test for unpaired data. Differences between means were considered significant when $p$ values were $p<0.05$. The statistical analysis was performed using GraphPad Prism 6.0.

\section{Results}

3.1. Angiogenesis Is Impaired in Synthetic Implants from iNOSDeficient Mice. The sponge matrix was well tolerated by animals of both wild-type and knockout groups. No signs of infection or rejection were observed in the implant location during the 14-day period of the experiment. Angiogenesis was assessed by evaluating implants hemoglobin content and blood vessels counting in H\&E-stained tissue sections, besides VEGF levels by ELISA. We observed reduced hemoglobin content (Figure 1(a)) and blood vessels count (Figures 1(b) and 1(d)) in the fibrovascular tissue at day 14 after sponge implantation in $\mathrm{iNOS}^{-/-}$mice when compared to the WT counterparts. In WT mice, the hemoglobin content $\left(\mu \mathrm{g} / \mathrm{mg}\right.$ wet tissue) was $2.52 \pm 0.12$ and, in $\mathrm{iNOS}^{-/-}$mice, it fell to $1.88 \pm 0.13(p<0.01)$. Corroborating these data, the measurement of the proangiogenic cytokine VEGF was also decreased in sponges from iNOS ${ }^{-/-}$mice, as shown in Figure 1(c). Altogether, these data suggest that sponge-induced angiogenesis is impaired in the absence of iNOS activity.

3.2. Leukocyte Infiltration Is Enhanced in Implants from iNOSDeficient Mice. The inflammatory component was determined by estimating the neutrophil and macrophage accumulation into the implants through assaying MPO and NAG enzyme activities, respectively, as well as measuring levels of cytokines (CXCL1, CCL2, TNF- $\alpha$, IL-10, and IFN- $\gamma$ ) in the implants. Oppositely to the reduced formation of new blood vessels in the newly formed fibrovascular tissue, we observed an enhancement in the leukocyte accumulation into implants of $_{\text {iNOS }}^{-/-}$mice when compared to WT, as assayed by MPO (Figure 2(a), $p<0.05$ ) and NAG activities (Figure 2(b), $p<0.05)$, suggesting increased infiltration of neutrophils and macrophages, respectively. The histopathology confirmed the increased leukocyte infiltration in implants from iNOS ${ }^{-/-}$ mice compared to WT mice (Figure 2(c)).

Interestingly, levels of the proinflammatory cytokine TNF- $\alpha$ (Figure 3(a), $p<0.05$ ) were decreased, while levels of the anti-inflammatory cytokine IL-10 (Figure 3(b), $p<$ $0.001)$ were increased in sponges from iNOS ${ }^{-/-}$mice when compared with WT mice. However, no differences were observed in CXCL1 (Figure 3(c)), CCL2 (Figure 3(d)), or IFN- $\gamma$ (Figure 3(e)) levels between both groups.
3.3. Collagen Deposition Is Increased in Implants from iNOS-Deficient Mice. Another important component of the fibrovascular tissue is the collagen deposition. Here, we estimated the content of collagen present in the implants by calculating the total area occupied by collagen $\left(\mu \mathrm{m}^{2}\right)$ in Picrosirius-red-stained histological sections evaluated under polarized light (Figure 4(a)). As shown in Figure 4(b), there was a significant increase in collagen deposition in implants from $\mathrm{iNOS}^{-/-}$mice when compared with WT mice $(p<$ $0.001)$, suggesting iNOS activity plays a role in adjusting collagen deposition during the inflammatory angiogenesis process induced by an implanted device.

\section{Discussion}

Nitric oxide (NO) is a diffusible gas involved in a vast number of biological processes that cover the cardiovascular, immune, and neural systems. Its production can be achieved via three known different NO synthase (NOS) enzymes isoforms: nNOS (neuronal; NOS1), iNOS (inducible; NOS2), and eNOS (endothelial; NOS3). Different from neuronal and endothelial isoforms, iNOS is regulated mainly on transcriptional level and is independent of intracellular calcium concentration [6]. Besides its important physiological roles in cardiovascular functions, especially in endothelium-dependent vasorelaxation, antiplatelet aggregation, and leukocyte adhesion, NO is a key mediator of inflammation ensuring antimicrobial and immunoregulatory functions as well as modulating by leukocyte recruitment [21]. The expression of iNOS, specifically, is involved in many inflammatory and neoplastic conditions [16]. More recently, the role of iNOS-induced NO during ischemia was also demonstrated [22]. All these physiopathological situations depend on new blood vessel formation to perpetuate or even cease.

In addition, considering the interest in implantation techniques and scaffolds for tissue engineering, it is worth mentioning that, as demonstrated by our group, levels of $\mathrm{NO}$ in polyether-polyurethane synthetic sponge implants are modulated by diverse angiogenesis-modifying treatments without, however, a definite correlation with inflammatory cell accumulation $[23,24]$. Moreover, iNOS activity seems to be crucial for adequate response to polypropylene implant integration in the peritoneum [25], although not necessary for the collagen deposition in synthetic PVA (polyvinylalcohol) sponges [26]. Nevertheless, the contribution of the iNOS isoform to the formation of a fibrovascular tissue in the polyether-polyurethane sponge implants was never investigated. Here, we studied the role of iNOS in different features of the foreign body response induced by that matrix implantation into the subcutaneous compartment of mice. Such implants are known to be infiltrated by a number of cells such as inflammatory cells, endothelial cells, and fibroblasts [19].

We demonstrated that iNOS expression may modulate the major concurrent components of the newly formed fibrovascular tissue (angiogenesis, inflammation, and collagen deposition) induced by sponge implantation. In the first set of results, we observed that angiogenesis and VEGF levels are reduced in the implants from iNOS ${ }^{-/-}$mice when 


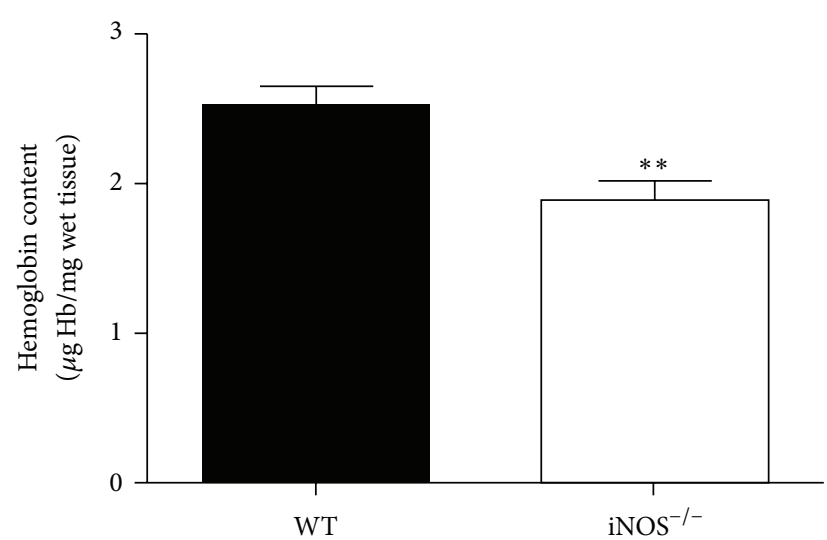

(a)

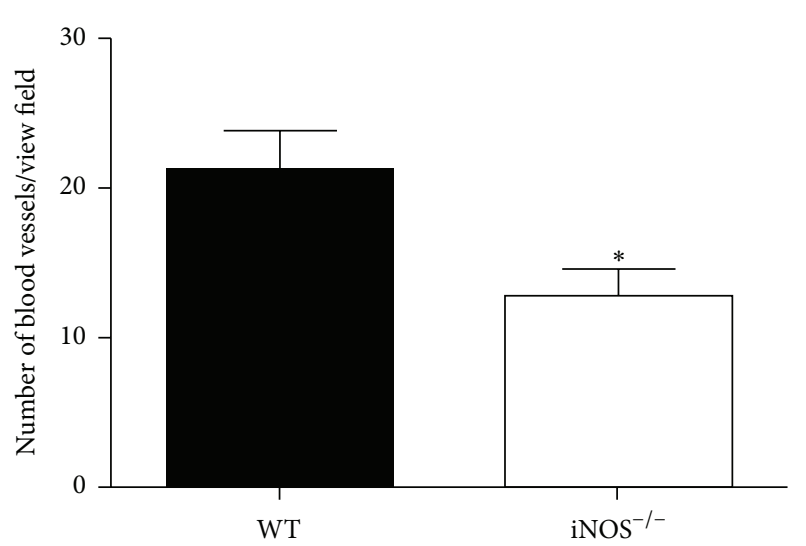

(b)

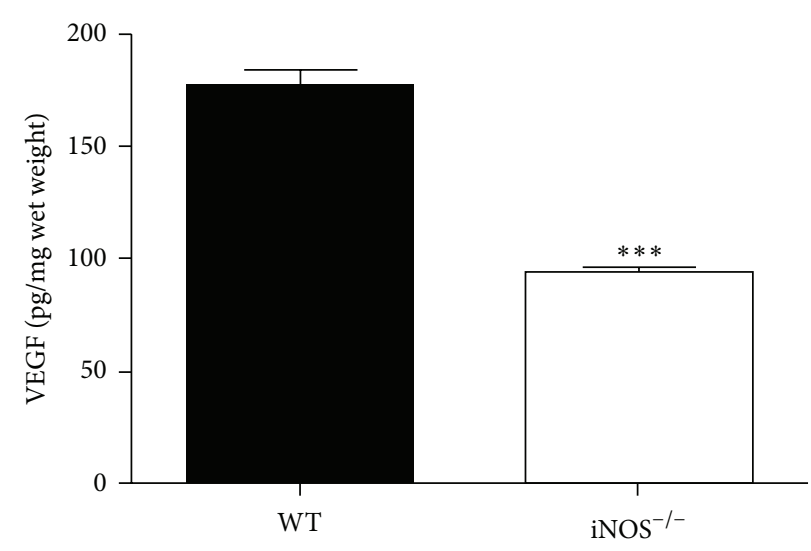

(c)

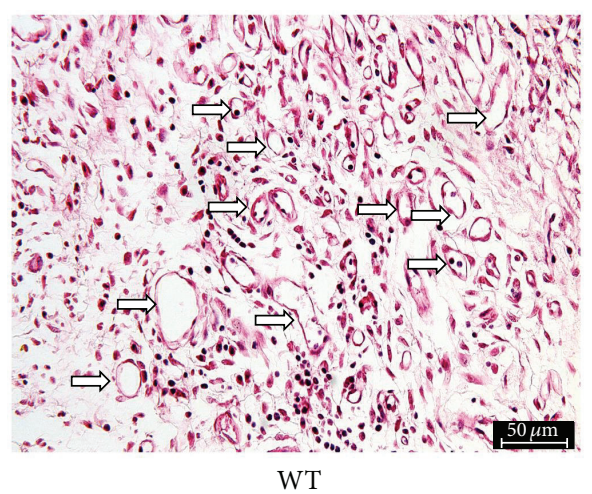

WT

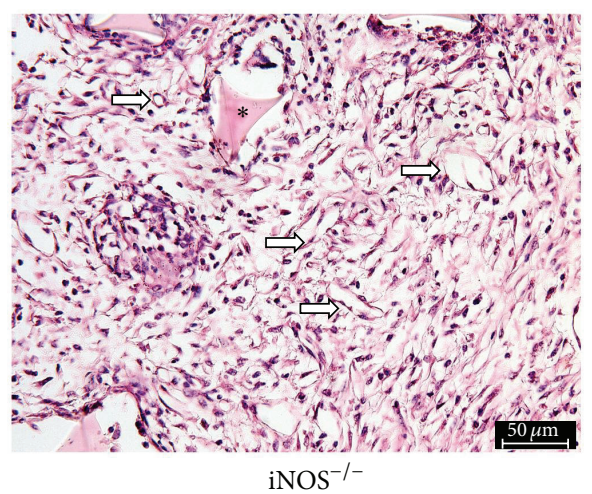

(d)

FIGURE 1: Impaired angiogenesis in implants from iNOS ${ }^{-1-}$ mice. Hemoglobin content (a). Blood vessels density (b). VEGF levels (c). Representative photomicrographs of H\&E-stained histological sections (d). The sponges were collected for analyses at day 14 after implantation. Values were represented as means ( \pm SEM) from groups of 8 animals each. ${ }^{* *} p<0.01,{ }^{* * *} p<0.001$ versus WT group (Student's $t$-test). Bar $50 \mu \mathrm{m}$. Asterisks indicate the synthetic matrix. White arrows indicate blood vessels.

compared to WT animals. Those data are in agreement with various reports in different in vitro and in vivo systems that demonstrated inhibition of iNOS results in decreased VEGF levels and consequently impaired angiogenesis [2729]. Indeed, the NO production by iNOS may, for instance, contribute to the release of VEGF [30], a growth factor known to be important to stimulate endothelial cell migration and the angiogenic process in vivo [31, 32]. VEGF, in turn, may stimulate eNOS activity resulting in further release of NO $[26,33,34]$. Therefore, our results suggest that impaired angiogenesis in the sponge implants from $\mathrm{iNOS}^{-/-}$mice may be attributed, at least partially, to the reduced NO-induced VEGF levels.

Angiogenesis and inflammation are interconnected processes in many pathophysiological conditions as well as in response to synthetic devices [35]. Here, we measured several 


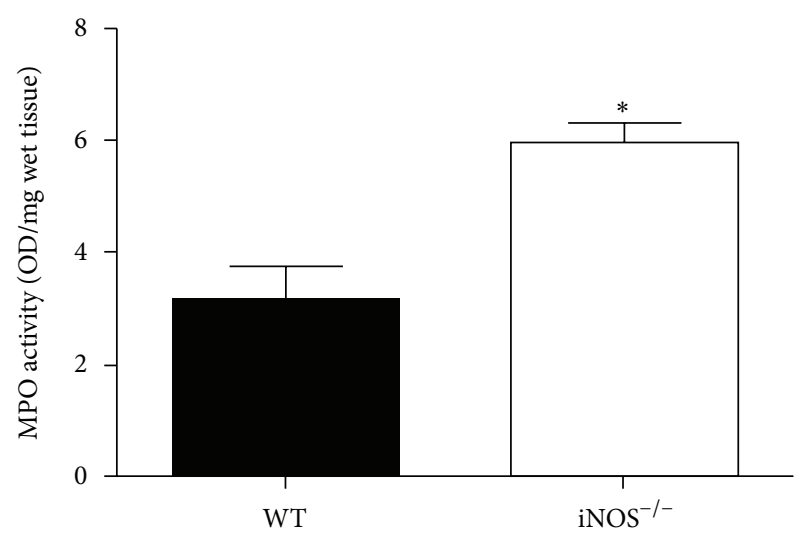

(a)

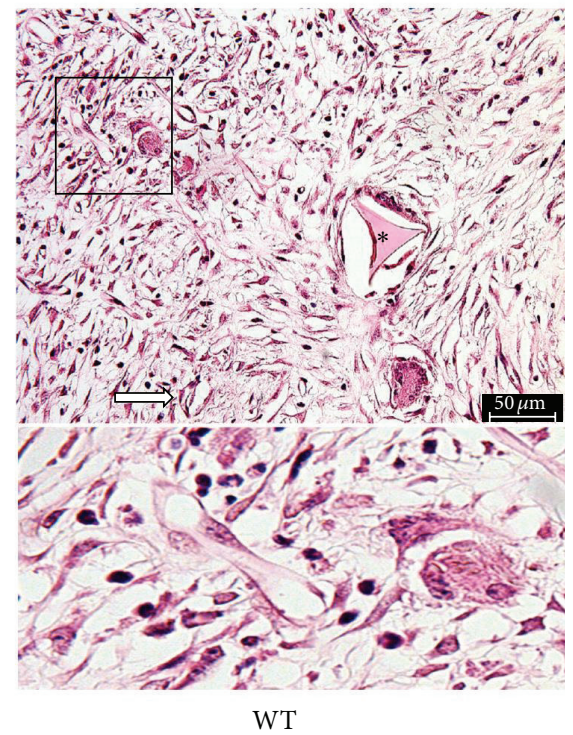

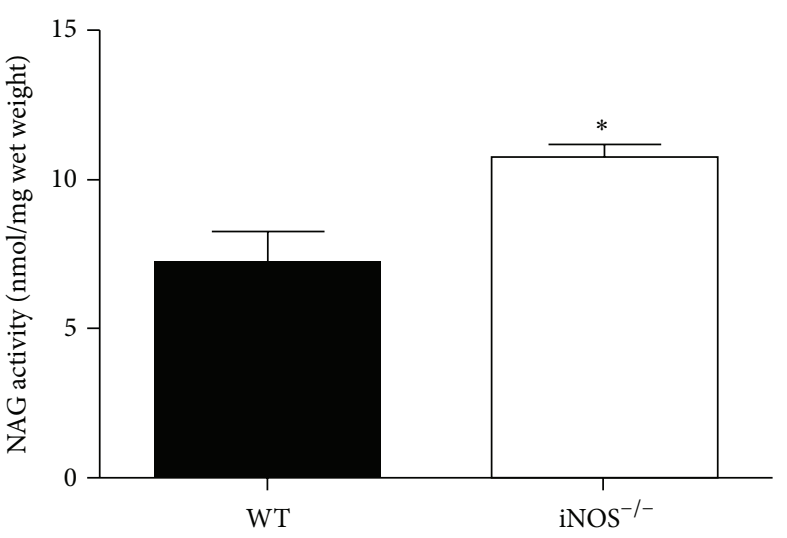

(b)

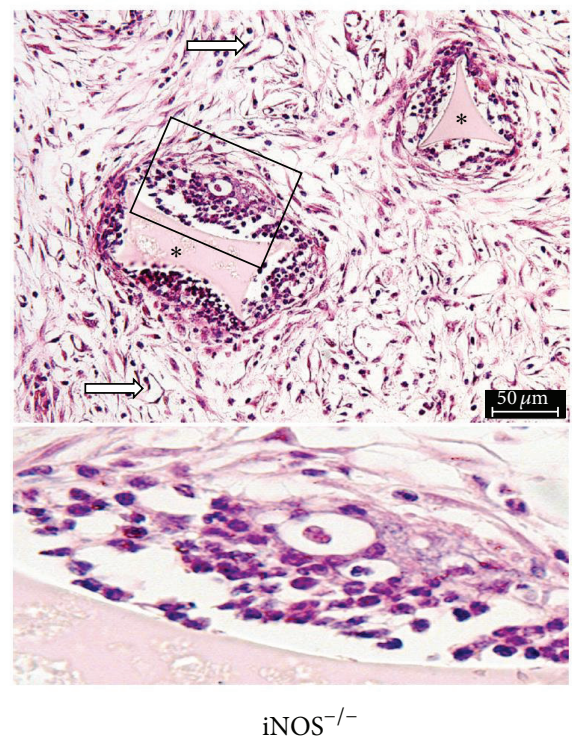

(c)

FIGURE 2: Increased leukocyte accumulation in implants from iNOS ${ }^{-1-}$ mice. The contents of neutrophils (a) and macrophages (b) recruited to the implant were determined by myeloperoxidase (MPO) and N-acetyl- $\beta$-D-glucosaminidase (NAG) activities, respectively. Representative photomicrographs of H\&E-stained histological sections (c). The sponges were collected at day 14 after implantation. Values were represented as means ( \pm SEM) from groups of 8 animals each. ${ }^{*} p<0.05$ versus WT group (Student's $t$-test). Bar $50 \mu \mathrm{m}$. Asterisks indicate the synthetic matrix. White arrows indicate blood vessels.

markers of inflammation (MPO and NAG activities and TNF- $\alpha$, CXCL1/KC, CCL2/MCP-1, IFN- $\gamma$, and IL-10 levels) in 14-day-old implants from WT and $\mathrm{iNOS}^{-/-}$animals. We observed that iNOS deficiency resulted in a prolonged inflammatory cell infiltration into the implants with both neutrophils and macrophages content being increased in sponges from iNOS ${ }^{-/-}$when compared to WT mice. However, no differences were observed in the levels of the respective chemoattractants CXCL1 or CCL2. In effect, there is a vast literature demonstrating that $\mathrm{NO}$ is a vascular protective molecule that prevents leukocyte adhesion to the endothelium and trafficking [36]. Likewise, there is evidence that iNOS-derived NO may modulate leukocyte and platelet functions that are involved in leukocyte recruitment to inflammatory site [37, 38]. Hickey et al. [39], using a LPS in vitro model, showed that iNOS-derived nitric oxide was able to reduce the adhesion of leukocytes under hydrodynamic flow conditions, providing a plausible explanation for the enhanced leucocyte recruitment observed in vivo in iNOS ${ }^{-1-}$ mice. Therefore, it is reasonable to speculate that the signaling pathways of the inflammatory chemokines CXCL1 and CCL2 are preserved at some extension in the implants from iNOS ${ }^{-/-}$mice and that the increased accumulation of inflammatory cells in the sponge from these animals may be attributed to the lack of iNOS-derived NO production, but not increase in the chemoattractant production.

Intriguingly, we found reduced levels of the proinflammatory cytokine TNF- $\alpha$ in the implants from iNOS ${ }^{-/-}$mice but increased levels of the anti-inflammatory cytokine IL10 , when compared with WT mice. In accordance, decreased production of proinflammatory cytokines in iNOS ${ }^{-1-}$ mice has been shown [40,41]. In addition, a compensatory 


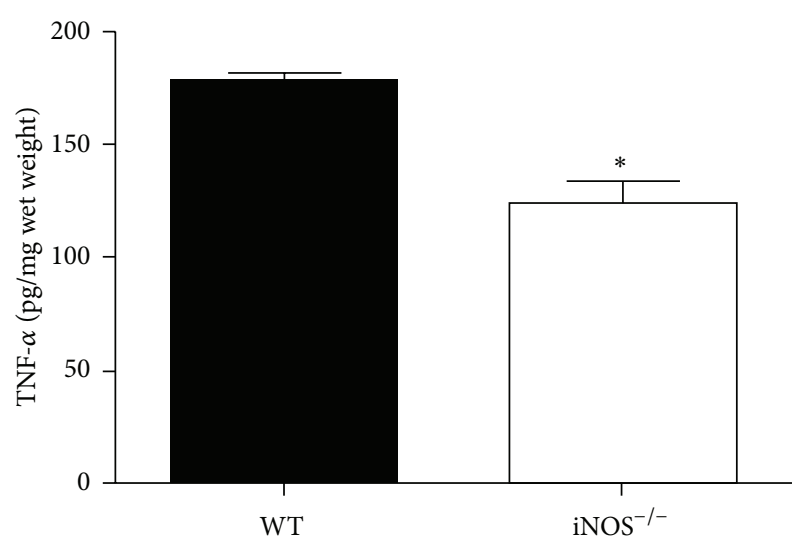

(a)

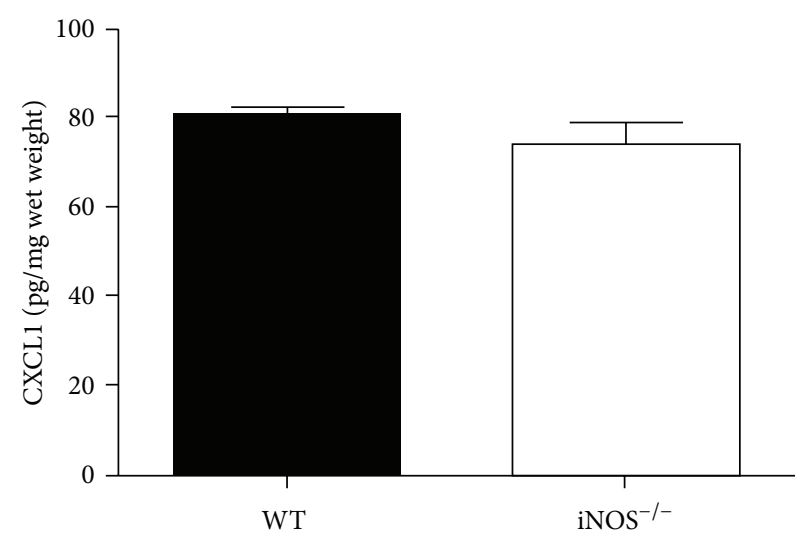

(c)

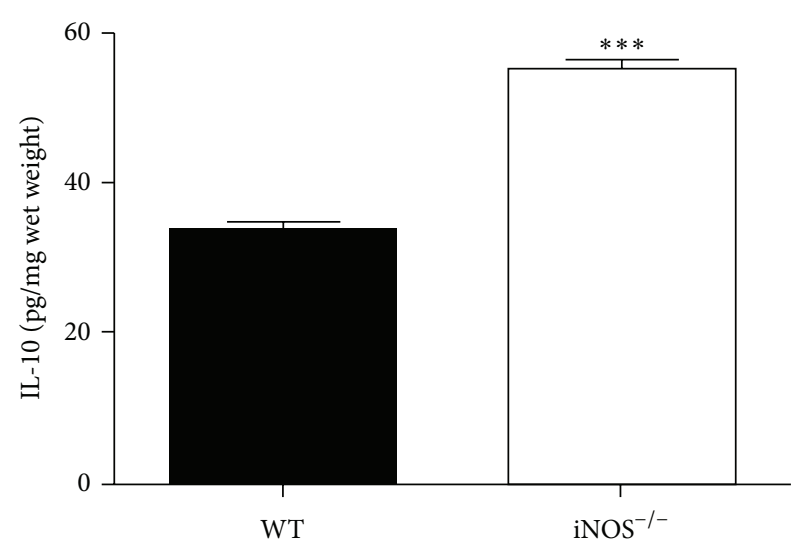

(b)

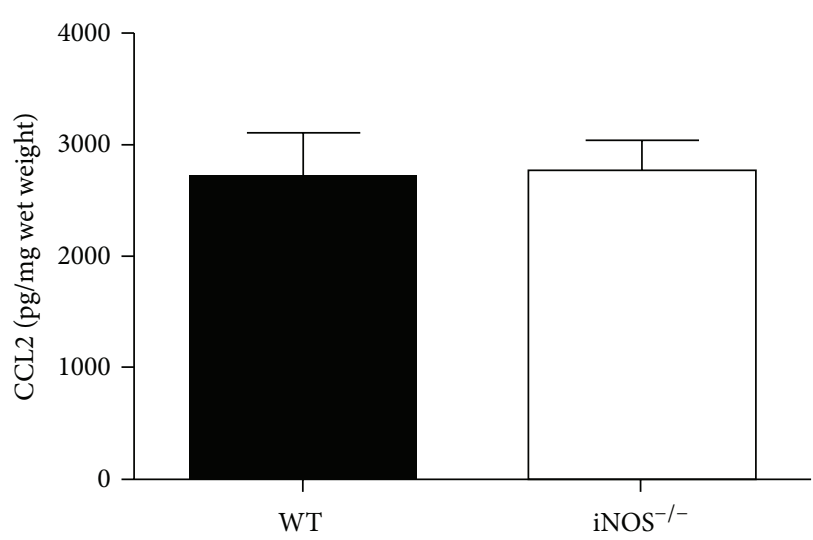

(d)

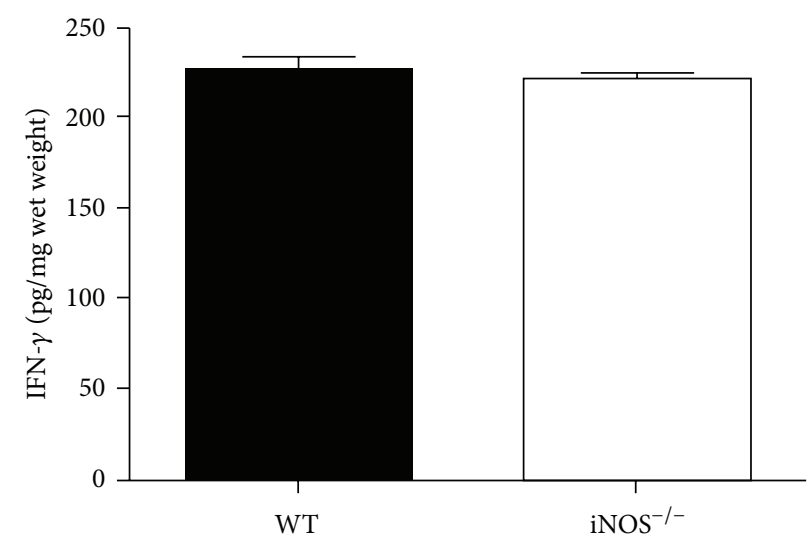

(e)

FIgURE 3: Modulation of cytokines, but not chemokines production, in implants from iNOS ${ }^{-/-}$mice. TNF- $\alpha$ (a), IL-10 (b), CXCL1/KC (c), CCL2/MCP-1 (d), and IFN- $\gamma$ (e) levels were evaluated by sandwich-type ELISA. The sponges were collected at day 14 after implantation. Values were represented as means $\left( \pm\right.$ SEM) from groups of 8 animals each. ${ }^{*} p<0.05,{ }^{* *} p<0.01$, and ${ }^{* * *} p<0.001$ versus WT group (Student's $t$-test).

overproduction of IL-10 may be observed in mice lacking the gene for the enzyme iNOS $[42,43]$. Of note, IL-10 is associated with an antiangiogenic effect by its ability to decrease VEGF levels [15] which, in turn, could partially help to explain the reduced levels of VEGF in the implants.

The collagen deposition during tissue remodeling is regulated by complex interactions of pro- and antifibrogenic proteins within the inflammatory tissue [44-46]. There are some pieces of evidence in the literature regarding the NO regulation of collagen deposition as, for example, increased collagen deposition observed in excisional wounds in $\mathrm{iNOS}^{-/-}$mice [12]. In addition, neutrophils and macrophages are well known to be involved in inflammation-induced fibrosis [47]. Therefore, the accumulation of inflammatory 


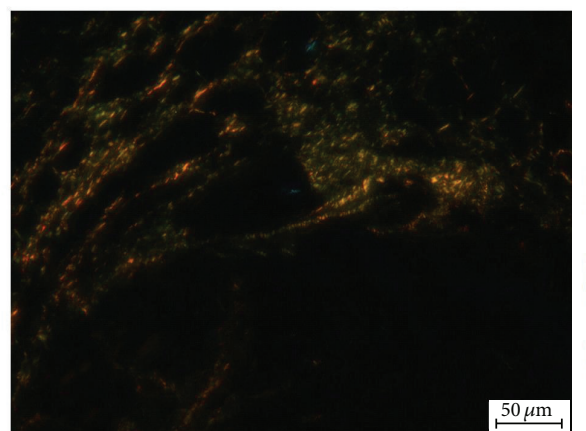

WT

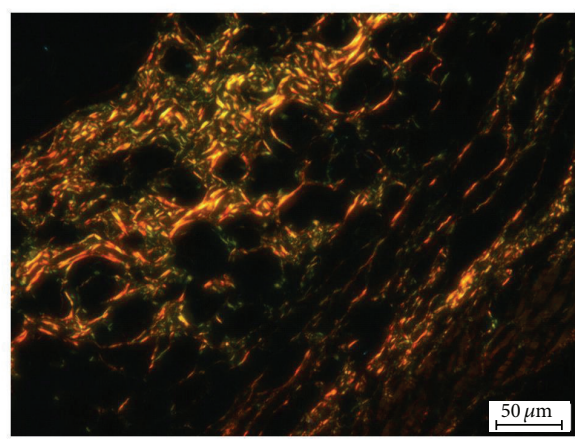

$\mathrm{iNOS}^{-/-}$

(a)

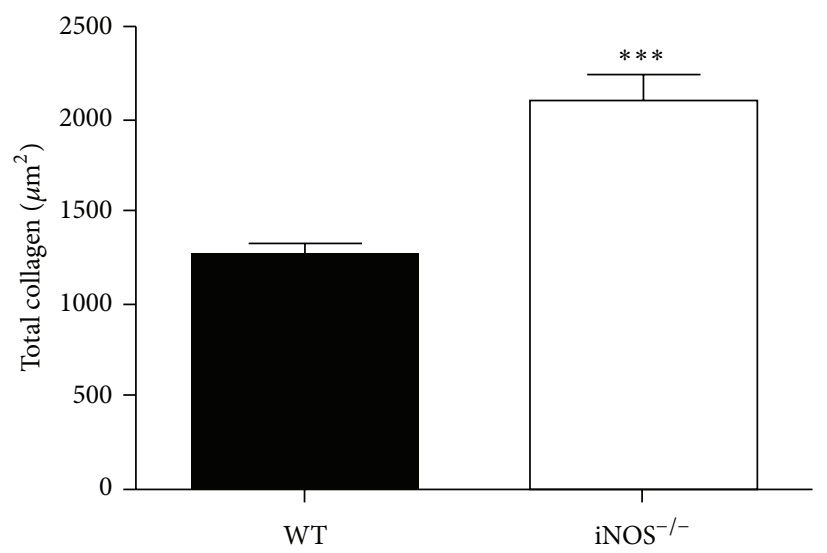

(b)

FIGURE 4: Enhanced collagen deposition in implants from iNOS ${ }^{-1-}$ mice. Representative photomicrographs of Picrosirius-red-stained sections (a) for collagen assessment under polarized light microscopy. Morphometric analysis of collagen content (b). The sponges were collected at day 14 after implantation. Values were represented as means $\left( \pm\right.$ SEM) from groups of 8 animals each. ${ }^{* * *} p<0.001$ versus WT group (Student's $t$-test). Bar $50 \mu \mathrm{m}$.

cells into the implants of iNOS-deficient mice could be one possible explanation of why there is an exaggerated fibrotic response in the implants from those animals. In fact, in the absence of iNOS activity, macrophages may acquire a nonclassical activated phenotype that may have profibrotic characteristics $[48,49]$ and then may account for the increased collagen deposition in the implants from iNOS-deficient mice. In addition, it is worth mentioning that although IL-10 is understood as an antifibrotic cytokine, it has been suggested that IL-10 cooperates with Th1 cytokines, such as IFN- $\gamma$ (and even TNF- $\alpha$ ), to suppress collagen deposition $[50,51]$. Interestingly, here, there were no changes in IFN- $\gamma$ levels, and TNF- $\alpha$ levels were even decreased, indicating a possible lack of the regulatory pathway for collagen deposition and, consequently, a supportive microenvironment for increasing fibrogenesis in the implants is endorsed in the absence of iNOS activity. Altogether, our data suggest that iNOS expression helps in controlling fibrosis in the sponge implants.

Overall, the absence of iNOS leads to increased foreign body reaction to implants in mice, observed as reduced angiogenesis and exacerbated inflammation and fibrosis in the synthetic implants. Therefore, the present work suggests a potential role for iNOS as a regulator of inflammatory angiogenesis during the fibrovascular tissue formation in sponge implants. These observations may provide relevant information for future insights in therapeutic strategies for conditions where inflammation and angiogenesis are associated as well as for the development of scaffolds for tissue engineering.
Abbreviations
CCL2: Chemokine (C-C motif) ligand 2
CCR 2: C-C chemokine receptor type 2
CXCL1: Chemokine (C-X-C motif) ligand 1
ELISA: Enzyme Linked Immunosorbent Assay
HTAB: Hexadecyltrimethylammonium bromide
IFN- $\gamma$ : Interferon gamma
IL-10: Interleukin 10
iNOS: Inducible nitric oxide synthase
KO: $\quad$ Knockout mice
MPO: Myeloperoxidase
NAG: $\quad$ N-Acetyl- $\beta$-D-glucosaminidase
NO: $\quad$ Nitric oxide
NOS: Nitric oxide synthase 
OD: Optic density

SEM: Standard error of the mean

TNF- $\alpha$ : Tumor necrosis factor- $\alpha$

VEGF: Vascular endothelial growth factor

WT: Wild-type.

\section{Conflict of Interests}

The authors declare that there is no conflict of interests.

\section{Acknowledgments}

The authors would like to thank Dr. Leda Quércia Vieira for the iNOS knockout animals and Valdinéria Borges and Ilma M. Souza for the technical support. This work was supported by Conselho Nacional de Pesquisa/CNPq, Coordenação de Aperfeiçoamento de Pessoal de Nível Superior/CAPES, and Fundação de Amparo à Pesquisa de Minas Gerais/FAPEMIG, Brazil. Luciola Silva Barcelos, Silvia Passos Andrade, and Mauro Martins Teixeira hold CNPq Research Fellowships.

\section{References}

[1] T. O. Socarrás, A. C. Vasconcelos, P. P. Campos et al., "Foreign body response to subcutaneous implants in diabetic rats," PLoS ONE, vol. 9, no. 11, Article ID el10945, 2014.

[2] S. P. Andrade, "Sponge implant model of angiogenesis," in Angiogenesis Protocols, vol. 46 of Methods in Molecular Medicine, pp. 77-86, 2001.

[3] M. J. Hickey, "Role of inducible nitric oxide synthase in the regulation of leucocyte recruitment," Clinical Science, vol. 100, no. 1, pp. 1-12, 2001.

[4] S. Y. Yoo and S. M. Kwon, "Angiogenesis and its therapeutic opportunities," Mediators of Inflammation, vol. 2013, Article ID 127170, 11 pages, 2013.

[5] M. A. Robinson, J. E. Baumgardner, and C. M. Otto, "Oxygendependent regulation of nitric oxide production by inducible nitric oxide synthase," Free Radical Biology and Medicine, vol. 51, no. 11, pp. 1952-1965, 2011.

[6] A. Pautz, J. Art, S. Hahn, S. Nowag, C. Voss, and H. Kleinert, "Regulation of the expression of inducible nitric oxide synthase," Nitric Oxide: Biology and Chemistry, vol. 23, no. 2, pp. 75-93, 2010.

[7] S. Kossmann, H. Hu, S. Steven et al., "Inflammatory monocytes determine endothelial nitric-oxide synthase uncoupling and nitro-oxidative stress induced by angiotensin II," The Journal of Biological Chemistry, vol. 289, no. 40, pp. 27540-27550, 2014.

[8] A. Türler, J. C. Kalff, B. A. Moore et al., "Leukocyte-derived inducible nitric oxide synthase mediates murine postoperative ileus," Annals of Surgery, vol. 244, no. 2, pp. 220-229, 2006.

[9] C. Raposo, A. K. D. S. Nunes, R. L. D. A. Luna, S. M. D. R. Araújo, M. A. Da Cruz-Höfling, and C. A. Peixoto, "Sildenafil (Viagra) protective effects on neuroinflammation: the role of iNOS/NO system in an inflammatory demyelination model," Mediators of Inflammation, vol. 2013, Article ID 321460, 11 pages, 2013.

[10] S. Heemskerk, R. Masereeuw, F. G. M. Russel, and P. Pickkers, "Selective iNOS inhibition for the treatment of sepsis-induced acute kidney injury," Nature Reviews Nephrology, vol. 5, no. 11, pp. 629-640, 2009.
[11] L. C. Chin, P. Kumar, J. A. Palmer et al., "The influence of nitric oxide synthase 2 on cutaneous wound angiogenesis," The British Journal of Dermatology, vol. 165, no. 6, pp. 1223-1235, 2011.

[12] D. Most, D. T. Efron, H. P. Shi, U. S. Tantry, and A. Barbul, "Characterization of incisional wound healing in inducible nitric oxide synthase knockout mice," Surgery, vol. 132, no. 5, pp. 866-876, 2002.

[13] A. J. Kane, J. E. Barker, G. M. Mitchell et al., "Inducible nitric oxide synthase (iNOS) activity promotes ischaemic skin flap survival," British Journal of Pharmacology, vol. 132, no. 8, pp. 1631-1638, 2001.

[14] L. Garcia-Bonilla, J. M. Moore, G. Racchumi et al., "Inducible nitric oxide synthase in neutrophils and endothelium contributes to ischemic brain injury in mice," The Journal of Immunology, vol. 193, no. 5, pp. 2531-2537, 2014.

[15] J.-S. Silvestre, Z. Mallat, M. Duriez et al., "Antiangiogenic effect of interleukin-10 in ischemia-induced angiogenesis in mice hindlimb," Circulation Research, vol. 87, no. 6, pp. 448-452, 2000.

[16] M. Lechner, P. Lirk, and J. Rieder, "Inducible nitric oxide synthase (iNOS) in tumor biology: the two sides of the same coin," Seminars in Cancer Biology, vol. 15, no. 4, pp. 277-289, 2005.

[17] S. P. Nichols, A. Koh, N. L. Brown et al., "The effect of nitric oxide surface flux on the foreign body response to subcutaneous implants," Biomaterials, vol. 33, no. 27, pp. 6305-6312, 2012.

[18] M. J. Reed, D. Eyman, and N. Karres, "Nitric oxide effects on the function of aged cells ex vivo and in vivo," In Vivo, vol. 22, no. 6, pp. 673-679, 2008.

[19] P. Cassini-Vieira, S. R. Deconte, T. C. Tomiosso et al., “DisBa-01 inhibits angiogenesis, inflammation and fibrogenesis of spongeinduced-fibrovascular tissue in mice," Toxicon, vol. 92, pp. 8189, 2014.

[20] L. S. Barcelos, A. M. Coelho, R. C. Russo et al., "Role of the chemokines CCL3/MIP-1 $\alpha$ and CCL5/RANTES in spongeinduced inflammatory angiogenesis in mice," Microvascular Research, vol. 78, no. 2, pp. 148-154, 2009.

[21] T. Persichini, O. Cantoni, H. Suzuki, and M. Colasanti, "Crosstalk between constitutive and inducible NO synthase: an update," Antioxidants \& Redox Signaling, vol. 8, no. 5-6, pp. 949954, 2006.

[22] M. Abu-Amara, S. Y. Yang, A. Seifalian, B. Davidson, and B. Fuller, "The nitric oxide pathway-evidence and mechanisms for protection against liver ischaemia reperfusion injury," Liver International, vol. 32, no. 4, pp. 531-543, 2012.

[23] S. Almeida, C. Cardoso, L. Orellano, A. Reis, L. Barcelos, and S. Andrade, "Natriuretic peptide clearance receptor ligand (C-ANP4-23 ) attenuates angiogenesis in a murine sponge implant model," Clinical and Experimental Pharmacology and Physiology, vol. 41, no. 9, pp. 691-697, 2014.

[24] F. A. Araújo, M. A. Rocha, L. S. A. Capettini et al., "3-Hydroxy3-methylglutaryl coenzyme A reductase inhibitor (fluvastatin) decreases inflammatory angiogenesis in mice," Acta Pathologica, Microbiologica, et Immunologica Scandinavica, vol. 121, no. 5, pp. 422-430, 2013.

[25] F. J. P. Souza-Pinto, A. I. S. Moretti, V. Cury, W. Marcondes, I. T. Velasco, and H. P. Souza, "Inducible nitric oxide synthase inhibition increases MMP-2 activity leading to imbalance between extracellular matrix deposition and degradation after polypropylene mesh implant," Journal of Biomedical Materials Research Part A, vol. 101, no. 5, pp. 1379-1387, 2013. 
[26] J. Dulak and A. Józkowicz, "Regulation of vascular endothelial growth factor synthesis by nitric oxide: facts and controversies," Antioxidants \& Redox Signaling, vol. 5, no. 1, pp. 123-132, 2003.

[27] J. M. Malone, G. M. Saed, M. P. Diamond, R. J. Sokol, and A. R. Munkarah, "The effects of the inhibition of inducible nitric oxide synthase on angiogenesis of epithelial ovarian cancer," American Journal of Obstetrics \& Gynecology, vol. 194, no. 4, pp. 1110-1116, 2006.

[28] R. P. Singh and R. Agarwal, "Inducible nitric oxide synthasevascular endothelial growth factor axis: a potential target to inhibit tumor angiogenesis by dietary agents," Current Cancer Drug Targets, vol. 7, no. 5, pp. 475-483, 2007.

[29] Y. Yang, T. Yu, Y.-J. Lian, R. Ma, S. Yang, and J. Y. Cho, "Nitric oxide synthase inhibitors: a review of patents from 2011 to the present," Expert Opinion on Therapeutic Patents, vol. 25, no. 1, pp. 49-68, 2015.

[30] S. Donnini and M. Ziche, "Constitutive and inducible nitric oxide synthase: role in angiogenesis," Antioxidants \& Redox Signaling, vol. 4, no. 5, pp. 817-823, 2002.

[31] P. Gerwins, E. Sköldenberg, and L. Claesson-Welsh, "Function of fibroblast growth factors and vascular endothelial growth factors and their receptors in angiogenesis," Critical Reviews in Oncology/Hematology, vol. 34, no. 3, pp. 185-194, 2000.

[32] A. Pyriochou, T. Vassilakopoulos, Z. Zhou, and A. Papapetropoulos, "cGMP-dependent and -independent angiogenesisrelated properties of nitric oxide," Life Sciences, vol. 81, no. 21-22, pp. 1549-1554, 2007.

[33] C. Li, L. Ruan, S. G. Sood, A. Papapetropoulos, D. Fulton, and R. C. Venema, "Role of eNOS phosphorylation at Ser-116 in regulation of eNOS activity in endothelial cells," Vascular Pharmacology, vol. 47, no. 5-6, pp. 257-264, 2007.

[34] B. K. Lal, S. Varma, P. J. Pappas, R. W. Hobson II, and W. N. Durán, "VEGF increases permeability of the endothelial cell monolayer by activation of $\mathrm{PKB} / \mathrm{akt}$, endothelial nitric-oxide synthase, and MAP kinase pathways," Microvascular Research, vol. 62, no. 3, pp. 252-262, 2001.

[35] Y.-W. Kim, X. Z. West, and T. V. Byzova, "Inflammation and oxidative stress in angiogenesis and vascular disease," Journal of Molecular Medicine, vol. 91, no. 3, pp. 323-328, 2013.

[36] P. Kubes, M. Suzuki, and D. N. Granger, "Nitric oxide: an endogenous modulator of leukocyte adhesion," Proceedings of the National Academy of Sciences of the United States of America, vol. 88, no. 11, pp. 4651-4655, 1991.

[37] T. Murohara, S. J. Parkinson, S. A. Waldman, and A. M. Lefer, "Inhibition of nitric oxide biosynthesis promotes P-selectin expression in platelets: role of protein kinase C," Arteriosclerosis, Thrombosis, and Vascular Biology, vol. 15, no. 11, pp. 2068-2075, 1995.

[38] M. J. Hickey, D. N. Granger, and P. Kubes, "Inducible nitric oxide synthase (iNOS) and regulation of leucocyte/endothelial cell interactions: studies in iNOS-deficient mice," Acta Physiologica Scandinavica, vol. 173, no. 1, pp. 119-126, 2001.

[39] M. J. Hickey, K. A. Sharkey, E. G. Sihota et al., "Inducible nitric oxide synthase (iNOS)-deficient mice have enhanced leukocyte-endotelium interactions in endotoxemia," The FASEB Journal, vol. 11, no. 12, pp. 955-964, 1997.

[40] E. McNeill, M. J. Crabtree, N. Sahgal et al., "Regulation of iNOS function and cellular redox state by macrophage Gchl reveals specific requirements for tetrahydrobiopterin in NRF2 activation," Free Radical Biology \& Medicine, vol. 79, pp. 206216, 2015.
[41] K. Abbas, J. Breton, A.-G. Planson et al., "Nitric oxide activates an Nrf2/sulfiredoxin antioxidant pathway in macrophages," Free Radical Biology \& Medicine, vol. 51, no. 1, pp. 107-114, 2011.

[42] G. Fritsche, M. Nairz, E. R. Werner, H. C. Barton, and G. Weiss, "Nrampl-functionality increases iNOS expression via repression of IL-10 formation," European Journal of Immunology, vol. 38, no. 11, pp. 3060-3067, 2008.

[43] D.-M. McCafferty, E. Sihota, M. Muscara, J. L. Wallace, K. A. Sharkey, and P. Kubes, "Spontaneously developing chronic colitis in IL-10/iNOS double-deficient mice," The American Journal of Physiology, vol. 279, no. 1, pp. G90-G99, 2000.

[44] T. A. Wynn, "Cellular and molecular mechanisms of fibrosis," The Journal of Pathology, vol. 214, no. 2, pp. 199-210, 2008.

[45] N. S. Greaves, K. J. Ashcroft, M. Baguneid, and A. Bayat, "Current understanding of molecular and cellular mechanisms in fibroplasia and angiogenesis during acute wound healing," Journal of Dermatological Science, vol. 72, no. 3, pp. 206-217, 2013.

[46] J. S. Duffield, M. Lupher, V. J. Thannickal, and T. A. Wynn, "Host responses in tissue repair and fibrosis," Annual Review of Pathology: Mechanisms of Disease, vol. 8, pp. 241-276, 2013.

[47] A. Pellicoro, P. Ramachandran, J. P. Iredale, and J. A. Fallowfield, "Liver fibrosis and repair: immune regulation of wound healing in a solid organ," Nature Reviews Immunology, vol. 14, no. 3, pp. 181-194, 2014.

[48] C. J. Ferrante and S. J. Leibovich, "Regulation of macrophage polarization and wound healing," Advances in Wound Care, vol. 1, no. 1, pp. 10-16, 2012.

[49] A. Sindrilaru and K. Scharffetter-Kochanek, "Disclosure of the culprits: macrophages-versatile regulators of wound healing," Advances in Wound Care, vol. 2, no. 7, pp. 357-368, 2013.

[50] R. Hoffmann, E. Wenzel, A. Huth et al., "Cytokine mRNA levels in alopecia areata before and after treatment with the contact allergen diphenylcyclopropenone," Journal of Investigative Dermatology, vol. 103, no. 4, pp. 530-533, 1994.

[51] P. Freyschmidt-Paul, K. J. McElwee, R. Happle et al., "Interleukin-10-deficient mice are less susceptible to the induction of alopecia areata," The Journal of Investigative Dermatology, vol. 119, no. 4, pp. 980-982, 2002. 


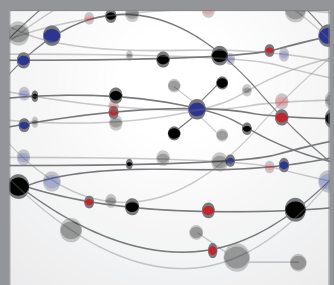

The Scientific World Journal
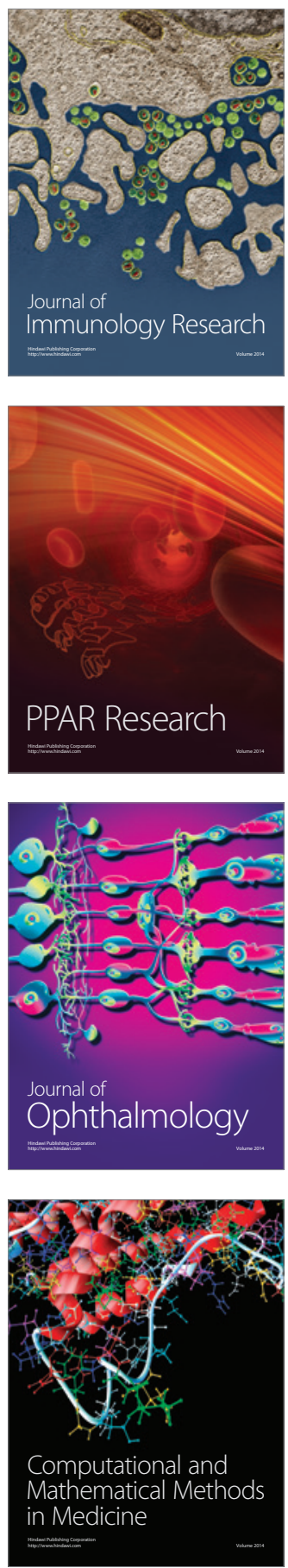

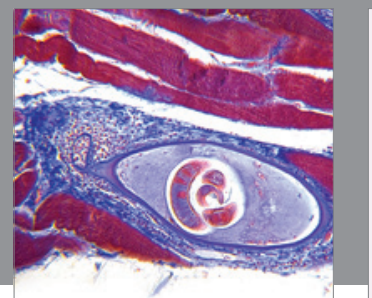

Gastroenterology

Research and Practice
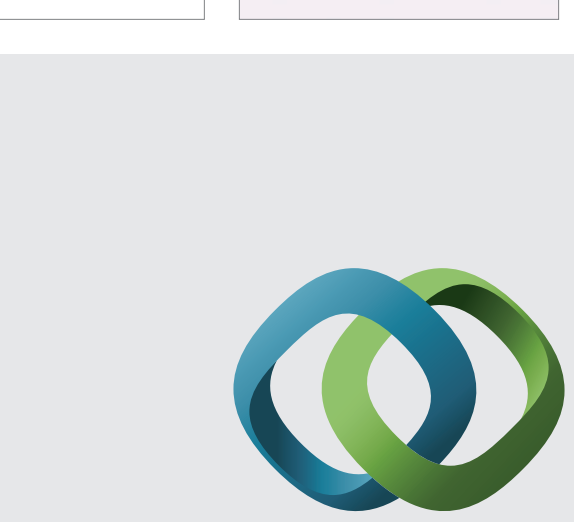

\section{Hindawi}

Submit your manuscripts at

http://www.hindawi.com
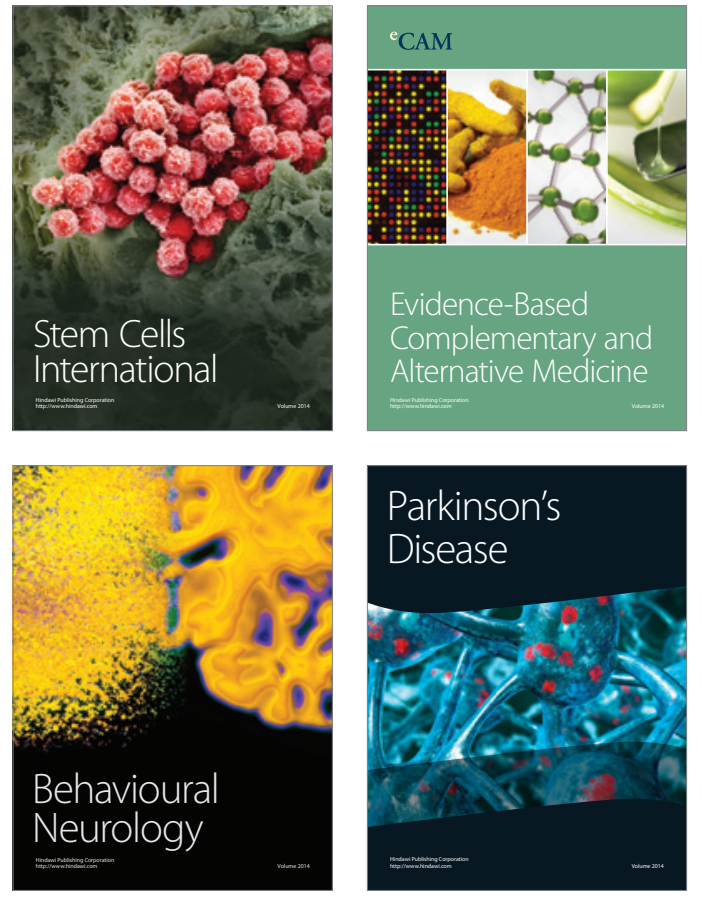
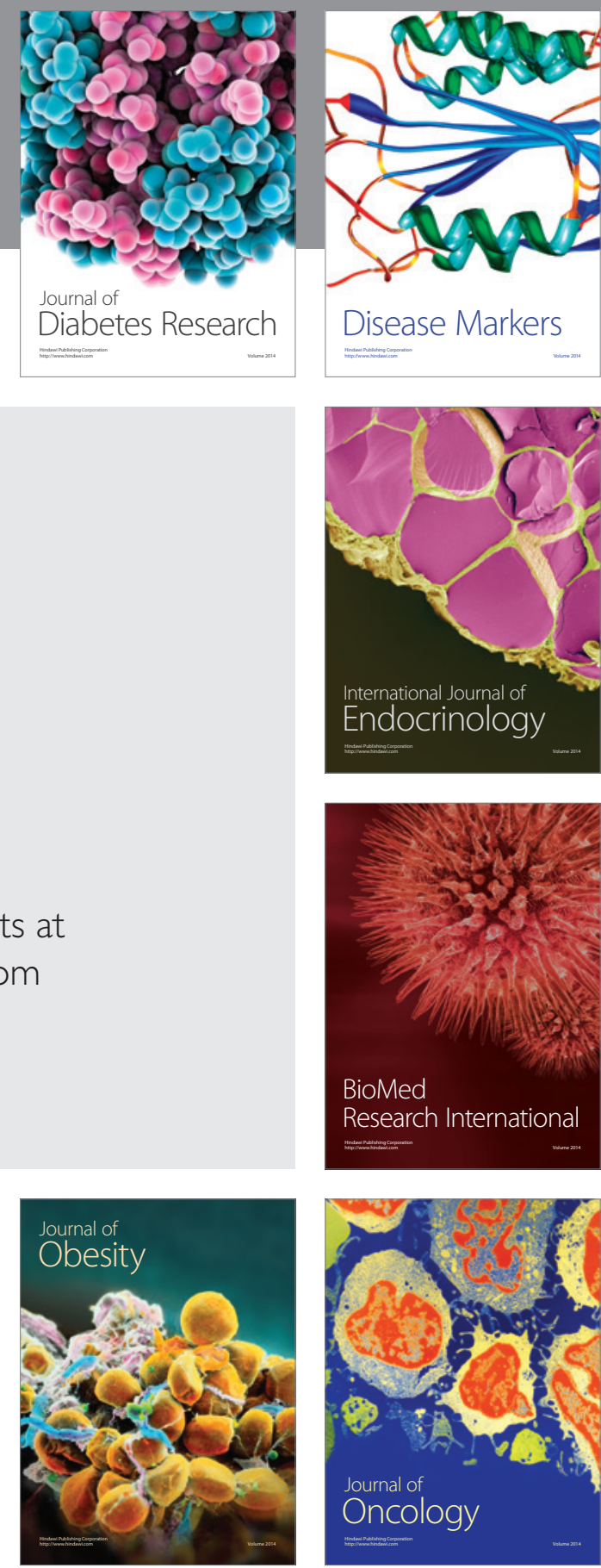

Disease Markers
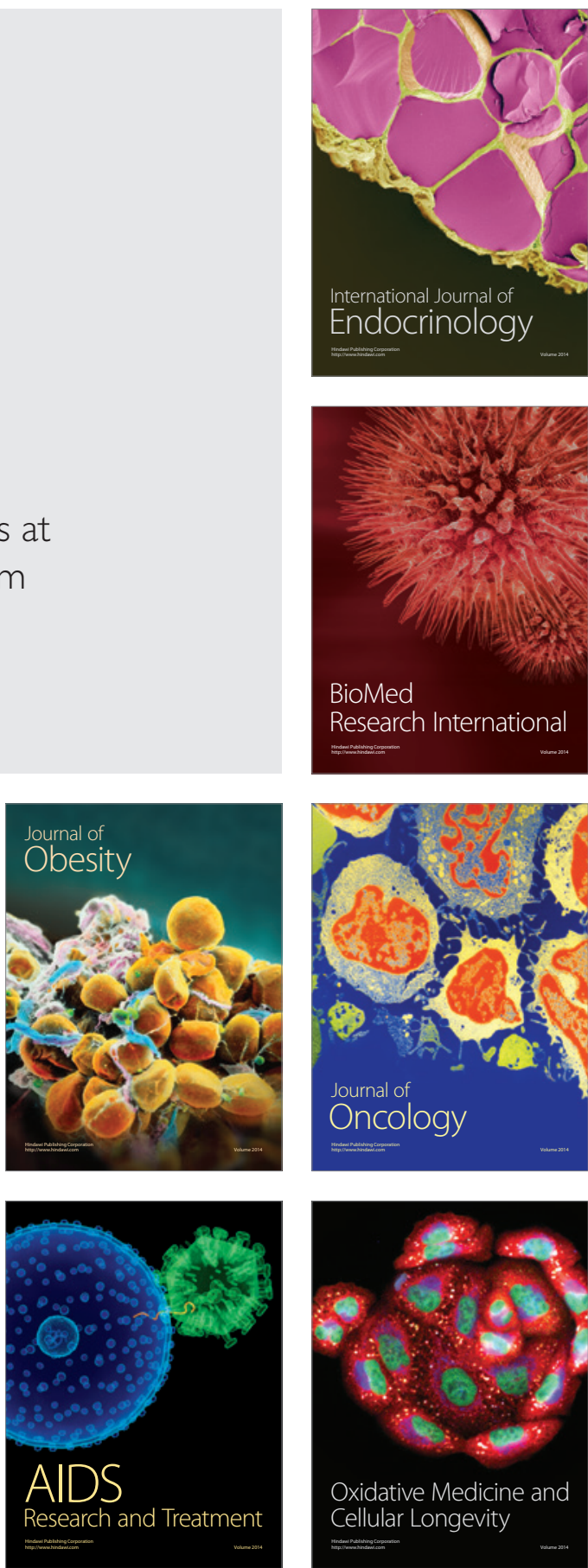